\title{
RECONHECIMENTO DE SENTENÇAS NO RUÍDO, EM CAMPO LIVRE, EM INDIVÍDUOS COM E SEM PERDA AUDITIVA
}

\author{
Sentence recognition thresholds, at the sound field, \\ for normal hearing subjects with and without hearing loss
}

Marília Oliveira Henriques ${ }^{(1)}$, Maristela Julio Costa ${ }^{(2)}$

\section{RESUMO}

Objetivo: determinar as relações sinal/ruído nas quais são obtidos os limiares de reconhecimento de sentenças no ruído (LRSR), em campo livre, para indivíduos com audição normal e para indivíduos com perda auditiva neurossensorial e comparar os resultados dos dois grupos pesquisados. Método: participaram do estudo 62 indivíduos adultos com idades entre 18 e 64 anos, sendo 32 normo-ouvintes e $30 \mathrm{com}$ perda de audição neurossensorial de grau leve a moderadamente severo, avaliados em cabina acusticamente tratada. Para a avaliação, as sentenças foram apresentadas em campo livre, na presença de um ruído competitivo, com uso do teste Lista de Sentenças em Português. Resultados: os LRSR dos indivíduos foram obtidos na relação sinal ruído de $-7,57 \mathrm{~dB}$ e para indivíduos com perda auditiva neurossensorial, em $-2,10 \mathrm{~dB}$. Ao comparar os resultados de ambos os grupos, a análise estatística apontou diferença estatisticamente significante entre os valores dos LRSR sendo $p<0,001$. Conclusão: as relações sinal-ruído em que são obtidos os limiares de reconhecimento de sentenças no ruído (LRSR), em campo-livre são iguais a $-7,57$ dB para indivíduos com audição normal e $-2,10 \mathrm{~dB}$ para indivíduos com perda auditiva neurossensorial, havendo diferença estatisticamente significante entre os resultados dos grupos estudados.

DESCRITORES: Audiologia; Testes de Discriminação da Fala; Ruído; Comunicação; Audiometria da Fala

\section{INTRODUÇÃO}

Os sons mais importantes transduzidos pela orelha são os que compõem os sons de fala, pois é por meio dela que a comunicação baseada na linguagem é transmitida 1.

A habilidade de um ouvinte em compreender a fala é afetada por muitos fatores, dentre eles o nível de apresentação do material, o tipo de apresentação

(1) Fonoaudióloga do corpo clínico do Serviço de Fonoaudiologia do Hospital Nossa Senhora da Conceição (HNSC), Porto Alegre, RS; Mestre em Distúrbios da Comunicação Humana pela Universidade Federal de Santa Maria - UFSM.

(2) Fonoaudióloga; Professora Adjunta do Curso de Fonoaudiologia da Universidade Federal de Santa Maria - UFSM; Doutora em Distúrbios da Comunicação Humana- Campo Fonoaudiológico pela Universidade Federal de São Paulo - UNIFESP - Escola Paulista de Medicina.

Conflito de interesses: inexistente e de resposta e as características do indivíduo, incluindo experiência de linguagem e condições do sistema auditivo 1.

Isso evidencia que apenas a obtenção dos limiares tonais e testes com palavras isoladas não permite uma análise mais abrangente da capacidade comunicativa de uma pessoa. Desta forma, para poder dimensionar a dificuldade auditiva do paciente avaliado, o audiologista precisa lançar mão de uma bateria de testes que não só deverão propiciar a identificação de uma perda auditiva, mas também a análise da compreensão da mensagem falada em situações próximas das condições de comunicação que o indivíduo encontra no seu dia-a-dia.

Se analisarmos as situações comunicativas de nosso cotidiano, vamos perceber que boa parte delas ocorre em ambientes cuja escuta é prejudicada pela presença de ruído competitivo. Esta condição é desfavorável para a inteligibilidade da 
fala, uma dificuldade freqüentemente relatada por pacientes na clínica audiológica, sendo que pacientes com os mesmos limiares tonais e com as mesmas habilidades auditivas de reconhecimento de fala no silêncio, podem ter habilidades de reconhecimento extremamente diferentes em ambientes ruidosos 1.

Por este motivo, testes de reconhecimento de sentenças no ruído são considerados uma ferramenta essencial na avaliação audiológica, por avaliarem as habilidades auditivas em condições que se aproximam das experiências auditivas cotidianas 2. Com a apresentação deste material em campo livre, pode-se ainda avaliar a compreensão da fala na condição binaural, que reflete a forma como se estabelece a comunicação diária 3.

No Brasil, o teste Listas de Sentenças em Português (LSP) 4 vem sendo aplicado na realização de diversos estudos desenvolvidos com a finalidade de obter informações sobre a real habilidade de reconhecimento da fala, em diferentes grupos de indivíduos 5-12. O teste proporciona precisão e objetividade para mensurar habilidades de reconhecimento de fala de um ouvinte, como reflexo de seu desempenho em situações auditivas realistas e seus achados, são de extrema importância para o diagnóstico clínico mais preciso.

Com base nos trabalhos já realizados com o LSP, em que foram estabelecidos valores de referência para o reconhecimento de fala para indivíduos normo-ouvintes 5,7 , verificou-se a necessidade de uma nova pesquisa que pudesse analisar a compreensão da fala em indivíduos com perda de audição neurossensorial, visto que a maioria dos indivíduos com este tipo de perda auditiva apresentam grande dificuldade na discriminação dos sons da fala, principalmente em ambientes ruidosos 13 .

Sendo assim, foi realizado o presente estudo, cujos objetivos foram determinar as relações sinalruído $(S / R)$ nas quais foram obtidos os limiares de reconhecimento de sentenças no ruído, em campo livre, para indivíduos com audição normal e para indivíduos com perda auditiva neurossensorial e comparar os resultados dos dois grupos pesquisados, podendo assim estimar o quanto os indivíduos que apresentam déficits auditivos terão sua habilidade comunicativa comprometida .

\section{MÉTODO}

O presente estudo foi desenvolvido no Ambulatório de Audiologia do Serviço de Atendimento Fonoaudiológico (SAF) da Universidade Federal de Santa Maria (UFSM).
Participaram da pesquisa somente os indivíduos que concordaram com a realização dos procedimentos necessários para a execução da pesquisa e que assinaram o Termo de Consentimento Livre e Esclarecido, após receberem maiores informações sobre o objetivo e a metodologia do estudo proposto.

Os critérios de inclusão adotados foram: idade entre 18 e 64 anos e limiares audiométricos dentro dos padrões de normalidade ou indicativos de perda auditiva neurossensorial de graus leve a moderadamente-severo ${ }^{14}$, pois o limite do audiômetro para saída de fala em campo-livre era de $65 \mathrm{~dB}$ NA.

Foram observados como critérios de exclusão a presença de alterações neurológicas, articulatórias e/ou de fluência verbal; a presença de rolha de cerume ou de outras alterações no meato acústico externo capazes de alterar o desempenho no teste; ausência de resposta ao teste Listas de Sentenças em Português ${ }^{4}$ e dificuldade para memorizar as sentenças. Também foram excluídos indivíduos normo-ouvintes que referiram não ouvir/compreender adequadamente ou que apresentaram queixa de zumbido.

No total, 81 indivíduos foram avaliados, sendo $36 \mathrm{com}$ limiares audiométricos dentro da normalidade e $45 \mathrm{com}$ perda auditiva. Destes, 62 foram selecionados para compor os grupos de estudo: 32 normo-ouvintes e $30 \mathrm{com}$ perda de audição neurossensorial, com idade entre 18 e 64 anos, sendo 25 homens e 37 mulheres.

As medidas deste estudo foram obtidas em cabina tratada acusticamente, utilizando-se um audiômetro digital de dois canais, marca Fonix, modelo FA-12, tipo I; fones auriculares tipo TDH-39 P, marca Telephonics, com um sistema de amplificação para audiometria em campo livre. As sentenças e o ruído foram apresentados por meio de um Compact Disc (CD) Player Digital da marca Toshiba, modelo 4149, acoplado ao audiômetro acima descrito.

Foi realizada previamente, por um profissional habilitado para este serviço, registrado no Inmetro $\mathrm{SP}$, a calibração do equipamento e as medidas em campo livre, foram obtidas em Nível de Pressão Sonora, usando a escala A do medidor, com respostas rápidas, por ser considerada aquela que mais se aproxima da resposta auditiva humana, além de ser a mais usada pela maioria dos pesquisadores nesta área 3,15.

Além disso, considerando as características do sinal de teste e da necessidade de manter sempre as mesmas condições acústicas do ambiente durante toda a pesquisa, as medidas em campo livre, foram monitoradas pela examinadora com o auxílio de um 
Medidor de Pressão Sonora Digital, da marca Radio Shack, para todos os pacientes avaliados.

Para estabelecer os parâmetros de calibração do canal das sentenças, foi utilizado como referência o tom puro presente na primeira faixa do $C D$, no canal um. O uso do tom puro foi necessário, pois a fala é um som complexo, que apresenta grande variação entre o som mais intenso e o menos intenso. Dessa forma, o uso de um som contínuo de referência garantiu que as condições de apresentação dos estímulos de fala fossem mantidas constantes.

Por sua vez, para o ruído, considerando que se trata de um som contínuo, a calibração foi realizada utilizando o próprio ruído como referência.

A saída de cada canal foi calibrada usandose o VU-meter do audiômetro. Tanto o tom puro, presente no canal um, quanto o ruído, presente no canal dois, foram colocados no nível zero.

É importante salientar que, segundo a autora do material ${ }^{4}$, após a sua publicação, foi verificado que as sentenças foram gravadas no $C D$ em uma intensidade média $7 \mathrm{~dB}$ abaixo da intensidade do tom puro e do ruído. Assim, para a análise dos dados, os $7 \mathrm{~dB}$ foram considerados e descontados dos níveis de intensidades médios calculados com os estímulos de fala para cada sentença apresentada. Por exemplo, se a média calculada foi $70 \mathrm{~dB}$ NPS (A), a intensidade real da fala apresentada em campo livre foi $63 \mathrm{~dB}$ NPS (A)

A avaliação dos indivíduos foi iniciada com a realização de uma anamnese, por meio de um questionário constituído por questões fechadas, as quais forneceram informações referentes a dados pessoais, nível de escolaridade, profissão, hábitos de vida diária, história otológica e queixas auditivas dos sujeitos estudados. Estas informações foram levantadas para dar suporte à avaliadora durante o exame e para pesquisar possíveis critérios de exclusão, sem finalidade de análise posterior.

A seguir, realizou-se a inspeção visual do meato acústico externo, com a finalidade de excluir da amostra indivíduos que apresentassem alterações que pudessem interferir nos resultados das avaliações propostas.

Posteriormente, os pacientes foram submetidos à Avaliação Audiológica Básica, constituída por: Audiometria Tonal Liminar por via aérea nas freqüências de 250 a $8.000 \mathrm{~Hz}$ e por via óssea nas freqüências de 500 a $4.000 \mathrm{~Hz}$; 16 ;pesquisa do Limiar de Reconhecimento de Fala (LRF) e Índice Percentual de Reconhecimento de Fala (IPRF) apresentados em fones 1 .

Após, os indivíduos selecionados foram avaliados para obtenção dos Limiares de Reconhecimento de Sentenças no Ruído (LRSR), em campo livre, de forma binaural. Para esta finalidade, foi aplicado o teste Listas de Sentenças em Português ${ }^{4}$, o qual é constituído por uma lista de 25 sentenças em Português brasileiro, sete listas com 10 sentenças cada uma e um ruído com espectro de fala. As sentenças e o ruído estão gravados em Compact Disc (CD), em canais independentes, e foram apresentados por meio de um CD Player acoplado ao audiômetro.

A aplicação do teste em campo livre foi realizada em ambiente acusticamente tratado, com o indivíduo posicionado a $1 \mathrm{~m}$ da fonte sonora, de frente para a mesma, inicialmente a $0^{\circ}-0^{\circ}$ azimute, ou seja, formando um ângulo de $0^{\circ}$ em relação aos planos horizontal e vertical ${ }^{4}$.

Foram utilizadas diferentes listas de sentenças, uma para cada condição de teste, a fim de eliminar a possibilidade de melhor desempenho devido à memorização das sentenças. O uso de listas diferentes não foi considerado como uma variável, pois as listas aplicadas nesta pesquisa são equivalentes entre si ${ }^{10}$. As sentenças utilizadas para obtenção dos LRSR encontram-se apresentadas na figura 1.

Para determinar o limiar de reconhecimento de sentenças no ruído (LRSR) dos indivíduos, foi utilizada a estratégia seqüencial ou adaptativa, ou ainda ascendente-descendente ${ }^{17}$, que permite determinar o limiar de reconhecimento de fala (LRF), que é o nível necessário para o indivíduo identificar, de forma correta, aproximadamente $50 \%$ dos estímulos de fala apresentados em uma determinada relação S/R. Neste procedimento, foi apresentado o estímulo de fala em uma determinada relação $S / R$. Se o indivíduo fosse capaz de reconhecer corretamente o estímulo de fala apresentado, a intensidade do mesmo era diminuída em intervalos pré-estabelecidos, caso contrário, sua intensidade era aumentada. Este procedimento foi repetido até o final da lista.

A literatura ${ }^{17}$ sugere que sejam utilizados intervalos de $4 \mathrm{~dB}$ até a primeira mudança no tipo de resposta e, posteriormente, os intervalos de apresentação dos estímulos sejam de $2 \mathrm{~dB}$ entre si até o final da lista. Entretanto, devido às possibilidades técnicas do equipamento disponível para a realização desta pesquisa, foram utilizados intervalos de apresentação das sentenças de $5 \mathrm{~dB}$ e $2,5 \mathrm{~dB}$, respectivamente.

Inicialmente, foi realizado um treinamento para familiarização do indivíduo com o teste. Para isso, foram apresentadas as sentenças de 1 a 10 da lista $1 \mathrm{~A}$, sem a presença de ruído competitivo. $A$ resposta solicitada ao indivíduo avaliado foi repetir cada sentença da maneira como havia compreendido, logo após a apresentação da mesma. Uma resposta só foi considerada correta quando o indivíduo repetiu, sem erros ou omissões, a sentença apresentada. 
É importante que estas medidas sejam iniciadas em uma condição na qual o indivíduo seja capaz de reconhecer corretamente a primeira sentença de cada lista, para que haja uma compreensão melhor da dinâmica do teste e também redução das variáveis. Assim sendo, para garantir esta condição, a intensidade inicial de apresentação das sentenças no silêncio, para a lista de treinamento, foi de $20 \mathrm{~dB}$ acima do LRF da melhor orelha.

A seguir, foram apresentadas, ainda para treinamento, as sentenças de 11 a 20 da lista $1 \mathrm{~A}$, com presença de um ruído competitivo constante a 65 dB NPS (A). Neste caso, a intensidade inicial de apresentação da lista foi $63 \mathrm{~dB}$ NPS $(\mathrm{A})$ e a do ruído de 65 dB NPS (A), o que resulta em uma relação $S / R$ inicial de $-2 \mathrm{~dB}$, que é considerada uma relação favorável para indivíduos com audição normal. Porém para os indivíduos com perda auditiva, isso não se confirma. Assim sendo, a etapa de treinamento serviu para familiarizar o indivíduo com o teste e para verificar a relação S/R inicial aproximada a fim de iniciar a pesquisa do LRSR, fazendo o ajuste necessário para que pudessem iniciar o teste em uma relação $S / R$ mais favorável.

As medidas obtidas no treinamento não foram consideradas na análise dos resultados da pesquisa.

Após o treinamento, foi apresentada a lista 1B, na presença de ruído competitivo e os níveis de apresentação das sentenças foram anotados, para depois ser calculada uma média a partir dos valores onde houve mudança no tipo de resposta. Este valor foi subtraído do nível do ruído apresentado (65 dB A), obtendo-se assim a relação $S / R$, na qual o indivíduo foi capaz de reconhecer em torno de $50 \%$ dos estímulos apresentados.

Este estudo foi registrado no Gabinete de Projetos sob número 018269 e aprovação no Comitê de Ética em Pesquisa do Centro de Ciências da Saúde, protocolo número 051/2005.

Finalmente, foi realizada a análise estatística dos resultados de ambos os grupos. Inicialmente foi aplicado o teste de Shapiro Wilk, que constatou que os dados em análise não seguiam uma distribuição normal. Por este motivo, optou-se por utilizar um teste não paramétrico (Kruskal-Wallis) para comparar os grupos entre si.

Para todos os testes aplicados, o nível de significância adotado foi $\mathrm{a}=0,05$.

\section{LISTA $1 A$}

1. Não posso perder o ônibus.

2. Vamos tomar um cafezinho.

3. Preciso ir ao médico.

4. A porta da frente está aberta.

5. A comida tinha muito sal.

6. Cheguei atrasado para a reunião.

7. Vamos conversar lá na sala.

8. Depois liga pra mim.

9. Esqueci de pagar a conta.

10. Os preços subiram ontem.

11. O jantar está na mesa.

12. As crianças estão brincando.

13. Choveu muito neste fim-de-semana.

14. Estou morrendo de saudade.

15. Olhe bem ao atravessar a rua.

16. Preciso pensar com calma.

17. Guardei o livro na primeira gaveta.

18. Hoje é meu dia de sorte.

19. O sol está muito quente.

20. Sua mãe acabou de sair de carro.

21. Ela vai viajar nas férias.

22. Não quero perder o avião.

23. Eu não conheci sua filha.

24. Ela precisa esperar na fila.

25. O banco fechou sua conta.

\section{LISTA 1B}

1. O avião já está atrasado.

2. O preço da roupa não subiu.

3. O jantar da sua mãe estava bom.

4. Esqueci de ir ao banco.

5. Ganhei um carro azul lindo.

6. Ela não está com muita pressa.

7. Avisei seu filho agora.

8. Tem que esperar na fila.

9. Elas foram almoçar mais tarde.

10. Não pude chegar na hora.

Figura 1 - Listas de Sentenças em Português utilizadas na avaliação 


\section{RESULTADOS}

A tabela 1 apresenta as médias aritméticas, desvios padrão e valores mínimo e máximo das relações S/R nas quais foram obtidos os LRSR para o grupo de indivíduos normo-ouvintes (grupo A) e para o grupo de indivíduos com perda de audição neurossensorial (grupo B), bem como o resultado do teste Kruskal-Wallis, que verificou a existência de diferença estatisticamente significante entre os LRSR dos grupos estudados.

Tabela 1 - Médias aritméticas, desvios padrão, valores mínimos e máximos das relações s/r para os grupos A e B e resultado do teste kruskal-Wallis

\begin{tabular}{ccccccc}
\hline GRUPO & N & MÉDIA & $\begin{array}{c}\text { DESVIO } \\
\text { PADRÃO }\end{array}$ & MíNIMO & MÁXIMO & Valor de $\mathbf{p}$ \\
\hline A & 32 & $-7,57$ & 1,60 & $-10,57$ & $-5,33$ & $\mathrm{p}<0,001^{*}$ \\
B & 30 & $-2,10$ & 2,87 & $-6,44$ & 3,25 & \\
\hline
\end{tabular}

Legenda 01: S/R- Sinal Ruído.

* diferença estatisticamente significante.

\section{DISCUSSÃO}

Os LRSR foram obtidos nas relações $S / R-7,57$ dB para indivíduos com audição normal e $-2,10$ $\mathrm{dB}$ para indivíduos com perda de audição neurossensorial de grau leve a moderadamente-severo, sendo a diferença entre os valores igual a 5,47 dB.

Foram encontrados, na literatura nacional e internacional, estudos realizados com o objetivo de obter LRSR com metodologias semelhantes à empregada na presente pesquisa ${ }^{18-21}$.

Um destes estudos verificou que, para que o grupo de indivíduos com perda de audição neurossensorial mantivesse o LRSR, são necessários 5 dB favoráveis na relação $S / R$. Enquanto indivíduos normais necessitaram de $-2 \mathrm{~dB}$ para obter $50 \%$ de acertos, os indivíduos com perda auditiva necessitaram de valores próximos $\mathrm{a}+3 \mathrm{~dB}$ para obter esse mesmo limiar ${ }^{18}$.

Posteriormente, outro experimento observou que indivíduos com perdas cocleares necessitavam de um aumento do sinal de 3 a $6 \mathrm{~dB}$ favoráveis na relação $S / R$ para manter o mesmo reconhecimento de fala no ruído que indivíduos normais ${ }^{19}$.

Em 1997, foi publicado um estudo onde foram apresentados os aumentos na relação S/R necessários para manter $50 \%$ da inteligibilidade em função da perda de audição. Segundo o autor, indivíduos com $30 \mathrm{~dB}$ de perda de audição podem necessitar de um aumento de $4 \mathrm{~dB}$ na relação $S / R$, enquanto que pessoas com $80 \mathrm{~dB}$ de perda auditiva necessitarão de $12 \mathrm{~dB}$ de aumento de relação $S / R$ para manter os mesmos $50 \%$. Ao observar os valores apresentados para perdas de 30 a $65 \mathrm{~dB}$ NA, que foi o grau máximo de perda auditiva avaliado nesta pesquisa, constata-se que, novamente, os valores obtidos foram confirmados, pois aproximam-se da média obtida neste autor pra estes graus de perda auditiva neurossensorial 20 .

No Brasil, em pesquisa realizada com o uso do teste, os LRSR foram obtidos nas relações $S / R$ de $-6,71 \mathrm{~dB}$ para indivíduos normais e $-1,15 \mathrm{~dB}$ para indivíduos com perda auditiva neurossensorial, com uma diferença de $5,56 \mathrm{~dB}$ entre os grupos ${ }^{21}$.

A partir da revisão de literatura realizada, foi possível verificar que os resultados do presente estudo diferem dos demais no que se refere aos valores absolutos, provavelmente em função de possíveis variáveis que podem ser encontradas na avaliação em campo livre, como tamanho da sala, condições acústicas, existência ou não de superfície refletora, nível de reverberação e, calibração. No entanto, a diferença observada entre os LRSR encontrados para os grupos avaliados foi similar.

Esta diferença entre os limiares dos grupos analisados já era esperada em função da redução na audibilidade ocasionada pela perda auditiva neurossensorial.

A fala é um sinal acústico cuja informação é transmitida por meio de mudança de relação de freqüência, intensidade e tempo. O sistema auditivo normal possui a capacidade inerente de identificar, processar e codificar essa informação. Assim, qualquer degradação na capacidade do sistema auditivo realizar essas funções pode levar a um declínio na habilidade de o ouvinte com deficiência auditiva entender a fala em certas situações de audição 22.

Vários aspectos associados à deficiência auditiva neurossensorial podem afetar a capacidade de um ouvinte com perda auditiva processar a fala. 
A forma mais evidente de ocorrência é a perda do acesso do sistema à informação fonológica devido à perda de sensibilidade do limiar - ou seja, partes ou talvez todo o sinal fonológico podem não ser audíveis. Quando a diminuição da audibilidade supera a redundância alta na fala (que certamente predomina no caso de situações auditivas favoráveis), será então observada uma diminuição no desempenho e identificação da fala, sendo que a maioria das pessoas que possuem perda auditiva de grau leve a moderado está apta a funcionar bem em circunstâncias nas quais as condições acústicas estão bem controladas - normalmente onde o ruído de fundo não é excessivo ou o ambiente excessivamente reverberante. No entanto, o motivo que leva à busca de um tratamento é a dificuldade de compreensão em meio ao ruído ${ }^{22}$.

Outro fator importante é o fato de que existem cinco déficits perceptuais associados à perda auditiva neurossensorial: elevação do limiar, redução da faixa dinâmica /sensação de intensidade, redução da seletividade de freqüência, redução da resolução temporal e processamento binaural alterado. Apenas a elevação do limiar é satisfatoriamente corrigida com instrumentos digitais 23 .

Sendo assim, na aplicação de teste LSP, ao elevar o nível de apresentação das sentenças, o paciente com perda de audição é favorecido em apenas um dos déficits associados à sua perda auditiva.

Pode, ainda, existir uma grande variação entre os limiares de reconhecimento de fala dos indivíduos com perda de audição, ou seja, nem sempre o indivíduo que tem limiares audiométricos piores terá um desempenho pior no teste. Essas diferenças variam conforme o local da lesão das células ciliadas da cóclea, sendo que o pior desempenho será dos indivíduos cujas células ciliadas internas da cóclea foram afetadas 20.

Em relação ao desempenho destes indivíduos em situações de reconhecimento de fala em situações do cotidiano, sabe-se que a diferença de $1 \mathrm{~dB}$ na relação S/R equivale a uma perda de $11,20 \%$ no reconhecimento de sentenças 24 . Logo, se estes indivíduos necessitam de cerca de $5 \mathrm{~dB}$ a mais para atingirem seus LRSR, entende-se que, em uma situação de escuta favorável apenas para normoouvintes, terão uma acentuada dificuldade para compreender a fala, pois a perda em termos de reconhecimento será superior a $50 \%$. Em variações mais amplas na relação $S / R$, essa variação pode ser de 0 a $100 \%$. Este dado é bastante relevante, tendo em vista que a maioria das situações de comunicação do cotidiano ocorrem em ambientes com condições de escuta desfavoráveis.

Diante desta realidade, destaca-se a importância da utilização dos dados obtidos nesta pesquisa na rotina clínica, pois o conhecimento dos valores apresentados pode contribuir para que o audiologista tenha maiores possibilidades de dimensionar a dificuldade de inteligibilidade de fala no ruído, que é um dos problemas relatados com mais freqüência por pessoas com perda auditiva neurossensorial e até mesmo por indivíduos normo-ouvintes, em alguns casos.

Ainda, ao observar a semelhança dos achados deste estudo com os resultados e comentários observados na literatura internacional, pode-se afirmar que o teste LSP mostrou-se um instrumento de avaliação relevante, podendo ser incorporado na rotina clínica para este tipo de avaliação.

Espera-se que esta pesquisa sirva como base para novos estudos, com diferentes populações, para que possam ser estabelecidos outros parâmetros de comparação e novos conhecimentos acerca do tema.

\section{CONCLUSÃO}

Ao término deste estudo, a apreciação crítica dos resultados permitiu concluir que as relações sinal-ruído nas quais são obtidos os limiares de reconhecimento de sentenças no ruído, em campolivre foram $-7,57 \mathrm{~dB}$ para indivíduos com audição normal e - 2,10 dB para indivíduos com perda de audição neurossensorial, havendo diferença estatisticamente significante entre os resultados destes grupos. Sendo assim, verifica-se que indivíduos com perda de audição neurossensorial necessitam de 5,47 dB mais favoráveis na relação $S / R$, para obter o mesmo desempenho de indivíduos normo-ouvintes. 


\section{ABSTRACT}

Purpose: to quantify the signal-to-noise ratios in which the sentence recognition in the noise (SRN) in the sound field can be obtained. The research aimed at subjects with normal hearing and subjects with sensorineural hearing loss and compared the results for both groups. Method: 62 subjects took part in the study, their age ranged from 18 to 64 years old, among these 32 had normal hearing and 30 had sensorineural hearing loss varying from mild to moderately severe degree. The exams were performed in an acoustical booth. The sentences were evaluated in the presence of a competitive noise, making use of the Sentences List in Portuguese. Results: for the group of people with normal hearing, the SRN were obtained at the $S / R$ ratio of $-7,57 \mathrm{~dB}$ and for the group with hearing loss the SRN were obtained at the $S / R$ ratio of $-2,10 \mathrm{~dB}$. The statistical analysis showed a statistically significant difference between the SRN among the groups $(p<0,001)$. Conclusions: the signal-to-noise ratios where the SRN obtained, in the sound field, and were $-7,57 \mathrm{~dB}$ for normal hearing subjects and $-2,10$ $\mathrm{dB}$ for subjects with sensorineural hearing loss; there was a statistically significant difference among the results of the studied groups.

KEYWORDS: Audiology; Speech Discrimination Tests; Noise; Communication; Audiometry; Speech

\section{REFERÊNCIAS}

1. Wilson RH, Strouse, AL. Audiometria com estímulos de fala. In: Musiek FE; Rintelmann WF. Perspectivas atuais em avaliação auditiva. 1 ed. Brasileira, 2001. p 21-56.

2. Theunissen M, Swanepoel DW, Hanekon J. Sentence recognition in noise: variables in compilation and interpretation of tests. Int $\mathrm{J}$ Audiol. 2009; 48 (11): 743-57.

3. Dubno JR, Ahlstrom JB, Horwitz AR. Binaural advantage for younger and older adults with normal hearing. J Speech Lang Hear Res. 2008; 51 (2): 539-56.

4. Costa MJ. Listas de sentenças em português: apresentação e estratégias de aplicação na audiologia. Santa Maria: Pallotti; 1998.

5. Miranda EC, Costa MJ. Reconhecimento de sentenças no silêncio e no ruído de indivíduos jovens adultos normo-ouvintes e campo livre. Fono Atual. 2006; 8 (35): 4-12.

6. Freitas CD, Lopes LFD, Costa MJ. Confiabilidade dos limiares de reconhecimento de sentenças no silêncio e no ruído. Rev Bras Otorrinolaringol. 2005; 71 (5): 624-30.

7. Henriques MO, Miranda EC, Costa MJ. Limiares de reconhecimento de sentenças no ruído, em campo livre: valores de referência para indivíduos adultos normo-ouvintes. Rev Bras Otorrinolaringol. 2008; 74(2): 188-92.

8. Aurélio NHS, Becker KT, Padilha C, Santos SN, Petry T, Costa MJ. Limiares de reconhecimento de sentenças no silêncio em campo livre versus limiares tonais em fone em indivíduos com perda auditiva coclear. Rev CEFAC. 2008; 10 (3): 378-84. 9. Aurélio NHS, Costa MJ. Curvas logoaudiométricas utilizando sentenças como estímulo. Rev CEFAC, ahead of print Epub Jan 29,2010.

10. Santos SN, Daniel RC, Costa MJ. Estudo da equivalência entre as listas de sentenças em português. Rev. CEFAC. 2009; 11 (4):673-80.

11. Santos SN, Petry T, Costa MJ. Índices percentuais de reconhecimento de sentenças no silêncio e no ruído: efeitos da aclimatização no indivíduo avaliado sem as próteses auditivas. Rev CEFAC, ahead of print Epub Apr 23, 2010.

12. Petry T, Santos SN, Costa MJ. Reconhecimento de fala segundo o tempo de uso da amplificação. Rev Bras Otorrinolarongol (Olnine), 2010; 76 (4): 462-8.

13. Henderson D, Salvi RJ, Boettcher FA, Clock $A E$. Correlatos neurofisiológicos da perda auditiva neurossensorial. In: Katz J .Tratado de Audiologia Clínica. 4 ed. São Paulo: Manole.2001. p 30-55.

14. Silman S, Silverman CA. Auditory Diagnosysm principles and applications. London: Singular Publishing Group; 1991.

15. Mendel LL. Objective and subjetctive hearing aid assessment outcomes. Am J Audiol. 2007; 16 (2): 118-29.

16. Wilber, LA. Audiometria tonal liminar: via aérea e via óssea. In: Musiek FE; Rintelmann WF. Perspectivas atuais em avaliação auditiva. 1 ed. Brasileira, 2001. p 1-20 .

17. Levitt $H$, Rabiner LR. Use of a sequential strategy in intelligibility testing. J Acoust Soc Am. 1967; 42 (3): 609-12. 
18. Gelfand SA, Ross L; Miller S. Sentence reception in noise from one versus two sources: effects of aging and hearing loss. J. Acoust. Soc. Am. 1988; 83 (1):248-56.

19. Plomp R. Noise, amplification and compression: considerations of three main issues in hearing aid design. Ear Hear. 1994; 15 (1): 2-12.

20. Kiliion M. Hearing Aids: past, present and future: moving toward normal conversations in noise. Britisch J. Audiol. 1997; 31:141-8.

21. Pagnossim DF, lorio MC, Costa MJ. Reconhecimento de sentenças em campo livre em indivíduos portadores de perda auditiva neurossensorial. J Brasil Fonoaudiol.2001; 2(2): 153 -9.
22. Gatehouse S, Robinson K. Testes fonológicos como mensurações do processo auditivo. In: Martin M. Logoaudiometria. 2 ed. São Paulo: Santos; 2005. p. 74-87.

23. Naylor G. Technical and audiological factors in the implementation use of digital signal processing hearing aids. Scand Audiol.1997; 26 (4): 223-9.

24. Henriques, MO. Limiares e índices percentuais de reconhecimento de sentenças no ruído, em campo-livre, para indivíduos adultos. [dissertação]. Santa Maria: 2006. Universidade Federal de Santa Maria.
http://dx.doi.org/10.1590/S1516-18462011005000024

RECEBIDO EM: 27/08/2010

ACEITO EM: 18/11/2010

Endereço para correspondência:

Marília Oliveira Henriques

Abram Goldsztein 82 /804

Porto Alegre - RS

CEP: 91450-155

E-mail: mariliahrq@yahoo.com.br 